Check for updates

Cite this: Phys. Chem. Chem. Phys., 2019, 21, 9145

Received 2nd February 2019,

Accepted 16th April 2019

DOI: $10.1039 / c 9 c p 00665 f$

rsc.li/pccp

The natural abundance of coiled coil (CC) motifs in the cytoskeleton and the extracellular matrix suggests that CCs play a crucial role in the bidirectional mechanobiochemical signaling between cells and the matrix. Their functional importance and structural simplicity has allowed the development of numerous applications, such as proteinorigami structures, drug delivery systems and biomaterials. With the goal of establishing CCs as nanomechanical building blocks, we investigated the importance of helix propensity and hydrophobic core packing on the mechanical stability of 4-heptad CC heterodimers. Using single-molecule force spectroscopy, we show that both parameters determine the force-induced dissociation in shear loading geometry; however, with different effects on the energy landscape. Decreasing the helix propensity lowers the transition barrier height, leading to a concomitant decrease in the distance to the transition state. In contrast, a less tightly packed hydrophobic core increases the distance to the transition state. We propose that this originates from a larger side chain dynamics, possible water intrusion at the interface as well as differences in solvation of the hydrophobic amino acids at the transition state. In conclusion, the different contributions of helix propensity and hydrophobic core packing need to be considered when tuning the mechanical properties of CCs for applications.

Coiled coils (CCs) are self-assembled, superhelical motifs that are naturally found in numerous proteins in the cytoskeleton and the extracellular matrix. ${ }^{1}$ CCs consist of two (or more) $\alpha$-helices, each characterized by a repetitive pattern of seven

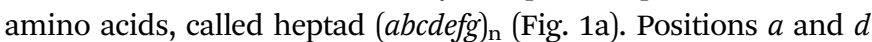
form the hydrophobic core of the superhelical structure; $e$ and $g$

\footnotetext{
${ }^{a}$ Max Planck Institute of Colloids and Interfaces, Mechano(bio)chemistry, Science-Park Potsdam Golm, 14424 Potsdam, Germany. E-mail: kerstin.blank@mpikg.mpg.de

${ }^{b}$ Max Planck Institute of Colloids and Interfaces, Department of Theory and Bio-Systems, Science-Park Potsdam Golm, 14424 Potsdam, Germany

${ }^{c}$ Freie Universität Berlin, Institute of Chemistry and Biochemistry - Organic Chemistry, Takustrasse 3, 14195 Berlin, Germany

${ }^{d}$ Max Planck Institute of Colloids and Interfaces, Department of Biomolecular Systems, Science-Park Potsdam Golm, 14424 Potsdam, Germany

$\dagger$ Electronic supplementary information (ESI) available. See DOI: 10.1039/c9cp00665f
}

a
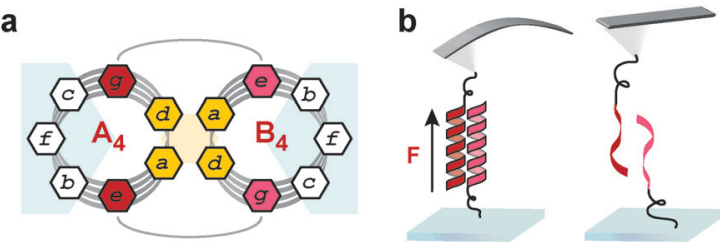

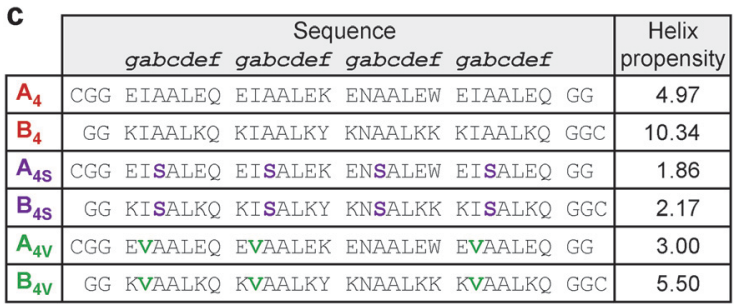

Fig. 1 Experimental design. (a) CC heptad pattern. (b) SMFS setup showing mechanical loading of a CC heterodimer in the shear geometry. (c) Sequences of the CCs used in this study. The terminal cysteines define the shear pulling geometry. The helix propensity of the CC-forming peptides was calculated using AGADIR. 4

are mostly charged amino acids, which participate in interhelical salt bridges; $b, c$ and $f$ are solvent-exposed, often polar amino acids, which contribute to the helix propensity of the individual helices. ${ }^{2,3}$

Utilizing this simple design, CCs serve as model systems for studying protein folding and stability. As a result, they are increasingly used as templates for protein design and sequences with a pre-determined thermodynamic stability can now be synthesized de novo. ${ }^{5-8}$ Such sequences find application in peptide-based hydrogels ${ }^{9-11}$ and protein origami structures ${ }^{12,13}$ as well as in biosensors ${ }^{14}$ and drug delivery systems. ${ }^{15}$ Considering their natural role as a mechanical scaffold, surprisingly little information is available about the sequence-structuremechanics relationship of CCs. With the goal of introducing CCs as nanomechanical building blocks, we have characterized three different CC heterodimers with atomic force microscope (AFM)-based single-molecule force spectroscopy (SMFS; Fig. 1b). We show that hydrophobic core packing and helix propensity 
affect the thermodynamic stability in similar ways; however, the underlying changes to the energy landscape are different.

Using the thermodynamically and mechanically wellcharacterized CC $\mathrm{A}_{4} \mathrm{~B}_{4}$ as the starting point (Fig. 1c), ${ }^{7,16}$ we used the standard rules of CC design ${ }^{2,5}$ to obtain one sequence with a reduced helix propensity and a second sequence with a different hydrophobic core packing. Throughout the manuscript, we define hydrophobic core packing as the combination of hydrophobicity and side chain packing of the amino acids in positions $a$ and $d$. To reduce the helix propensity, Ala in position $b$ was substituted with Ser in all heptads ${ }^{17}\left(\mathrm{~A}_{4 \mathrm{~S}} \mathrm{~B}_{4 \mathrm{~S}}\right.$; Fig. 1c). Hydrophobic core packing was altered using another $\beta$-branched amino acid in position $a$ (Val instead of Ile; $\mathrm{A}_{4 \mathrm{~V}} \mathrm{~B}_{4 \mathrm{~V}}$ ). The Asn in the third heptad was not replaced to maintain heterospecificity. ${ }^{18,19}$ For $\mathrm{A}_{4 \mathrm{~V}} \mathrm{~B}_{4 \mathrm{~V}}$, it needs to be considered that this substitution also decreases the helix propensity, which is lower for Val than for Ile (Fig. 1c). To define the points of force application, Cys was introduced at the desired termini for coupling the CC to the surface and the AFM cantilever. For A-peptides, Cys was located at the N-terminus, while it was placed at the C-terminus of B-peptides, thus establishing a shear pulling geometry (Fig. 1b).

To validate the design and to compare mechanical and thermodynamic stability, the CCs were first investigated with circular dichroism (CD) spectroscopy. Wavelength scans, showing an $\alpha$-helical signature with minima at $208 \mathrm{~nm}$ and $222 \mathrm{~nm}$ and thermal denaturation experiments are displayed in Fig. S1 (ESI $\dagger$ ). As expected, $\mathrm{A}_{4} \mathrm{~B}_{4}$ shows the highest melting temperature $T_{\mathrm{m}}$, while $\mathrm{A}_{4 \mathrm{~S}} \mathrm{~B}_{4 \mathrm{~S}}$ and $\mathrm{A}_{4 \mathrm{~V}} \mathrm{~B}_{4 \mathrm{~V}}$ were significantly destabilized (Table 1). The corresponding free energy difference between the folded (F) and the unfolded $(\mathrm{U})$ state $\left(\Delta G_{\mathrm{F}-\mathrm{U}}^{\circ}\right)$ was obtained from van't Hoff plots (Fig. S2, ESI $\dagger$ ). In comparison to $\mathrm{A}_{4} \mathrm{~B}_{4}$, both modified CCs also show a lower $\Delta G_{\mathrm{F}-\mathrm{U}}^{\circ}$, which follows a similar trend compared to other CCs reported in the literature. 5,20

To address the question whether the thermodynamic and mechanical stabilities are correlated and how the respective substitutions affect the energy landscape, SMFS was carried out. The B-peptide was immobilized to the cantilever, while the corresponding A-peptide was immobilized to the surface (Fig. S3, ESI $\dagger$ ). The CC forming peptides cooperatively fold and associate when the cantilever is in contact with the surface. When retracting the cantilever, the CC experiences a steadily increasing force until it ruptures, yielding two unfolded monomers (Fig. 1b). At a retract speed of $400 \mathrm{~nm} \mathrm{~s}^{-1}$, both $\mathrm{A}_{4 \mathrm{~S}} \mathrm{~B}_{4 \mathrm{~S}}$ and $\mathrm{A}_{4 \mathrm{~V}} \mathrm{~B}_{4 \mathrm{~V}}$ are mechanically less stable than $\mathrm{A}_{4} \mathrm{~B}_{4}$ (Fig. 2a). At this retract speed, the most probable rupture forces decrease $20 \mathrm{pN}$ and $15 \mathrm{pN}$ for $\mathrm{A}_{4 \mathrm{~S}} \mathrm{~B}_{4 \mathrm{~S}}$ and $\mathrm{A}_{4 \mathrm{~V}} \mathrm{~B}_{4 \mathrm{~V}}$, respectively.
This provides a first indication that the substitutions also lower the mechanical stability of the CCs.

Subsequent dynamic SMFS, performed over a range of loading rates $(r=\mathrm{d} F / \mathrm{d} t)^{21}$ from approximately $15 \mathrm{pN} \mathrm{s}^{-1}$ to $11000 \mathrm{pN} \mathrm{s}^{-1}$, revealed that both modified CCs possess a lower mechanical stability over the complete range of loading rates (Fig. S4-S6, ESI $\dagger$ ); however, their dependence on the loading rate is different. Fitting the data to the Bell-Evans model ${ }^{21}$ (Fig. 2b) allows for obtaining more detailed information about the energy landscape of the CC two-state system (folded CC vs. random coil peptides): $k_{\text {off }}$, the force-free dissociation rate, and $\Delta x_{\mathrm{F}-\mathrm{TS}}$, the distance from the folded to the transition state (TS). Using the Arrhenius equation, also the barrier height between the folded and the transition state $\left(\Delta G_{\mathrm{F}-\mathrm{TS}}\right)$ can be calculated, provided that the Arrhenius pre-factor is known. Here, we use an Arrhenius pre-factor of $5 \times 10^{8} \mathrm{~s}^{-1}$, which was estimated for the dimeric GCN4 leucine zipper. ${ }^{22}$ Table 1 summarizes the obtained fit values, as well as the calculated $\Delta G_{\mathrm{F}-\mathrm{TS}}$ values. In addition to the results shown in Table 1 , where the mean \pm standard error of the mean (SEM) was calculated from three independent experiments, a Peacock test ${ }^{23}$ was performed to determine if the modified CCs $\mathrm{A}_{4 \mathrm{~S}} \mathrm{~B}_{4 \mathrm{~S}}$ and $\mathrm{A}_{4 \mathrm{~V}} \mathrm{~B}_{4 \mathrm{~V}}$ are significantly different from the reference sequence $A_{4} B_{4}$. This test shows $p$-values smaller than 0.01 (see $\mathrm{ESI} \dagger$ for details).

Comparing the $k_{\text {off }}$ values presented in Table 1 shows that both modifications $\left(\mathrm{A}_{4 \mathrm{~S}} \mathrm{~B}_{4 \mathrm{~S}}\right.$ and $\left.\mathrm{A}_{4 \mathrm{~V}} \mathrm{~B}_{4 \mathrm{~V}}\right)$ lower the height of the transition state barrier with respect to the reference sequence $\mathrm{A}_{4} \mathrm{~B}_{4}$ (higher $k_{\text {off }}$ and lower $\Delta G_{\mathrm{F}-\mathrm{TS}}$ ). This lower barrier height is correlated with the different thermodynamic stabilities (Table 1) and helix propensities of the three CCs (Fig. 1c). This suggests that a reduced helix propensity lowers the barrier height, thereby affecting both the thermodynamic and mechanical stability of the CCs. Interestingly, the $\Delta x_{\mathrm{F}-\mathrm{TS}}$ values do not correlate with the thermodynamic stabilities. The Ala-Ser modification $\left(\mathrm{A}_{4 \mathrm{~S}} \mathrm{~B}_{4 \mathrm{~S}}\right)$ reduces $\Delta x_{\mathrm{F}-\mathrm{TS}}$, whereas the Ile-Val modification $\left(\mathrm{A}_{4 \mathrm{~V}} \mathrm{~B}_{4 \mathrm{~V}}\right)$ increases $\Delta x_{\mathrm{F}-\mathrm{TS}}$. This suggests that modifications in the solvent-exposed residues affect the energy landscape of the $\mathrm{CC}$ interaction differently when compared to hydrophobic core modifications.

In contrast to cooperative dissociation and unfolding upon increasing temperature, CCs respond to an applied axial stretching force in three phases. ${ }^{16,24-26}$ Initially, the force increases linearly with extension and the helices remain intact (phase I). At a strain of 10-20\%, the individual helices start uncoiling at an almost constant force (phase II). In long CCs, the force increases sharply after the helices are uncoiled and the resulting structure is extended further (phase III). For CCs

Table 1 Thermodynamic and kinetic parameters obtained with CD thermal denaturation experiments and SMFS at $25{ }^{\circ} \mathrm{C}(298 \mathrm{~K})$

\begin{tabular}{|c|c|c|c|c|c|c|}
\hline & $T_{\mathrm{m}}\left({ }^{\circ} \mathrm{C}\right)$ & $\Delta G_{\mathrm{F}-\mathrm{U}}^{\circ}\left(k_{\mathrm{B}} T\right)$ & $F^{a}(\mathrm{pN})$ & $\Delta x_{\mathrm{F}-\mathrm{TS}}(\mathrm{nm})$ & $k_{\text {off }}\left(\mathrm{s}^{-1}\right)$ & $\Delta G_{\mathrm{F}-\mathrm{TS}}\left(k_{\mathrm{B}} T\right)$ \\
\hline $\mathrm{A}_{4} \mathrm{~B}_{4}$ & $77.0 \pm 0.3$ & $14.2 \pm 0.3$ & $43.1 \pm 0.2$ & $1.3 \pm 0.2$ & $(3.2 \pm 2.1) \times 10^{-4}$ & $29.2 \pm 1.4$ \\
\hline $\mathrm{A}_{4 \mathrm{~S}} \mathrm{~B}_{4 \mathrm{~S}}$ & $54.3 \pm 0.3$ & $5.3 \pm 0.2$ & $23.6 \pm 4.8$ & $0.9 \pm 0.1$ & $(2.8 \pm 1.1) \times 10^{-1}$ & $21.3 \pm 0.2$ \\
\hline $\mathrm{A}_{4 \mathrm{~V}} \mathrm{~B}_{4 \mathrm{~V}}$ & $59.0 \pm 0.6$ & $7.1 \pm 0.6$ & $28.2 \pm 0.1$ & $1.7 \pm 0.0$ & $(2.4 \pm 1.7) \times 10^{-3}$ & $26.6 \pm 0.7$ \\
\hline
\end{tabular}

${ }^{a}$ Most probable rupture force $F$ determined at a retract speed of $400 \mathrm{~nm} \mathrm{~s}^{-1}$. All values are depicted as mean \pm standard error of the mean (SEM). 

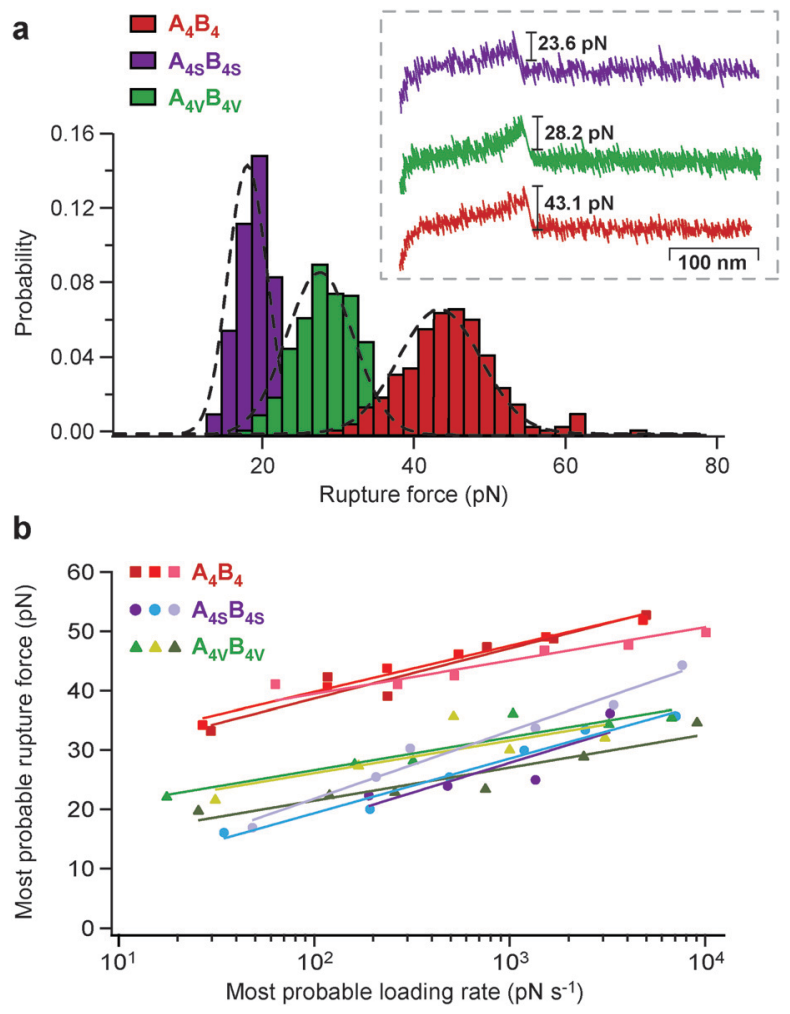

Fig. 2 Single-molecule force spectroscopy. (a) Representative rupture force histograms obtained at a retract speed of $400 \mathrm{~nm} \mathrm{~s}^{-1}$, with $n=284\left(A_{4} B_{4}\right)$, $n=243\left(\mathrm{~A}_{4 \mathrm{~S}} \mathrm{~B}_{4 \mathrm{~S}}\right)$ and $n=420\left(\mathrm{~A}_{4 \mathrm{~V}} \mathrm{~B}_{4 \mathrm{~V}}\right)$. The dashed lines show Gaussian fits, applied to extract the most probable rupture forces. The inset shows representative force-extension curves for each CC. (b) Dynamic SMFS plot. Each CC was measured in triplicate using a different cantilever and surface (different shades of the same colour). The solid lines represent fits to the Bell-Evans model.

with a length of $\leq 4$ heptads loaded in the shear geometry, the CC chains separate in phase I or just at the transition to phase II. ${ }^{16}$ This is a direct result of the force-induced chain separation mechanism. At loading rates typically used in SMFS, the applied force causes the uncoiling of helical structure at the points of force application. This, in turn, destabilizes the CC thermodynamically and facilitates the subsequent dissociation of the CC chains (uncoiling-assisted dissociation). This mechanism allows for explaining the effects of helix propensity and hydrophobic core packing on CC shearing.

When mechanically loaded in shear geometry, the hydrogen bonds stabilizing the individual helices are aligned parallel to the force vector, whereas the hydrophobic side chains are arranged almost perpendicularly. The torsional angles and helical propensities of the individual amino acids, which are responsible for maintaining stable hydrogen bonds in the helices, ${ }^{17,27,28}$ are thus critically determining the resistance of CCs to shear forces. For CCs with a lower overall helix propensity less force is required to uncoil the individual helices. In addition, a lower helix propensity is correlated with a lower thermodynamic stability. Uncoiling of only small parts of helical structure thus destabilizes an already less stable CC further, an effect that was observed earlier when decreasing CC length. ${ }^{16}$ Assuming that the hydrophobic core is not altered, this suggests that chain separation occurs at smaller extensions. In the case of $\mathrm{A}_{4 \mathrm{~S}} \mathrm{~B}_{4 \mathrm{~S}}$, a higher $k_{\text {off }}$ value is thus accompanied by a shorter $\Delta x_{\mathrm{F}-\mathrm{TS}}$. This result is in line with the observation that artificial constraints, which stabilize the helices against uncoiling, lead to an increase in the forces required for chain separation. ${ }^{3,11,29}$

Following this line of argumentation, $\Delta x_{\mathrm{F}-\mathrm{TS}}$ for $\mathrm{A}_{4 \mathrm{~V}} \mathrm{~B}_{4 \mathrm{~V}}$ is expected to lie in between the values obtained for $\mathrm{A}_{4 \mathrm{~S}} \mathrm{~B}_{4 \mathrm{~S}}$ and $\mathrm{A}_{4} \mathrm{~B}_{4}$; however, $\mathrm{A}_{4 \mathrm{~V}} \mathrm{~B}_{4 \mathrm{~V}}$ shows an increase in $\Delta x_{\mathrm{F}-\mathrm{TS}}$. This suggests that substituting Ile with Val does not only affect the helix propensity. We propose that the increased $\Delta x_{\mathrm{F}-\mathrm{TS}}$ originates from the combined effect of side chain hydrophobicity and packing. A reduced packing is expected to increase side chain dynamics at the hydrophobic interface, thus facilitating the rearrangement of the Val side chains in response to the applied force. In other words, a less densely packed hydrophobic core may allow the relative displacement of the helices prior to or during helix uncoiling. At the same time, changes in side chain hydrophobicity affect the interaction of the CC structure with water. Shear deformation may lead to water intrusion at the interface (wetting), followed by force-induced helix uncoiling. Alternatively, the helices may unfold without significant wetting. Independent of the presence of wetting, helix unfolding exposes hydrophobic amino acids to solvent, which need to be hydrated (solvation). ${ }^{20,30-33}$ While both wetting and solvation are primarily determined by side chain hydrophobicity, also the local environment ${ }^{31,32,34,35}$ and protein flexibility ${ }^{36}$ are expected to play important roles. As the two-state energy landscape model condenses the contribution of all these factors onto a single reaction coordinate (Fig. 3), it remains an open question which factor(s) determine the observed change in $\Delta x_{\mathrm{F}-\mathrm{TS}}$.

An increase in $\Delta x_{\mathrm{F}-\mathrm{TS}}$ has also been observed when introducing destabilizing substitutions in the hydrophobic core of the globular proteins GB1 and protein L. ${ }^{37,38}$ This effect, termed mechanical softening, was absent for the titin 127 domain, however, where mechanical unfolding is determined by a number of key hydrogen bonds. ${ }^{39,40}$ This suggests that

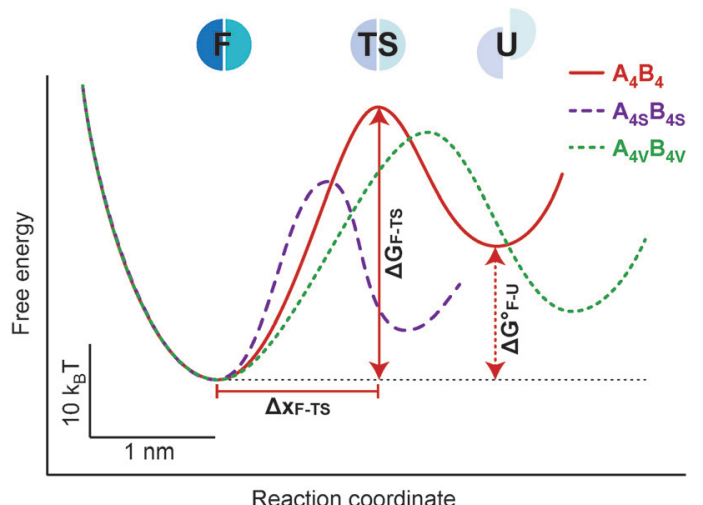

Fig. 3 Energy landscape of the CCs. The horizontal line represents the distance from the folded (F) to the transition state (TS) $\left(\Delta x_{F-T S}\right)$, while the vertical solid arrow represents the transition barrier height $\left(\Delta G_{\mathrm{F}-T S}\right)$. The dotted arrow shows the energy difference between the folded and the unfolded state $\left(\Delta G_{\mathrm{F}-\mathrm{U}}^{\circ}\right)$. 
substitutions in the hydrophobic core only affect the energy landscape parameters when positioned at a mechanically loaded interface. Clearly, CCs represent an attractive model system to dissect the different contributions of hydrophobicity and side chain packing to the mechanics of hydrophobic interfaces, e.g. via temperature-dependent SMFS experiments.

In summary, the combination of helix propensity and hydrophobic core packing determines the mechanical stability of CCs; however, with different effects on the energy landscape (Fig. 3). Whereas a reduced helix propensity decreases both the barrier height and the distance to the transition state, an increase in the transition state distance is obtained when decreasing the hydrophobic core packing. Clearly, both parameters can be utilized when designing CCs with controlled mechanical properties for future applications. Most interestingly, these parameters can be used to obtain CCs with similar thermodynamic stabilities that possess a different dynamic response to an externally applied force. Such systems will find application as molecular force sensors ${ }^{41}$ or as physical hydrogel crosslinks, which show a pre-defined response to mechanical deformation.

\section{Conflicts of interest}

There are no conflicts to declare.

\section{Acknowledgements}

The authors thank Isabell Tunn, Alberto Sanz de Leon and Ana Vila Verde for inspiring discussions. This work was funded by the Max Planck Society and the International Max Planck Research School (IMPRS) on Multiscale Bio-Systems. Open Access funding provided by the Max Planck Society.

\section{References}

1 A. N. Lupas and J. Bassler, Trends Biochem. Sci., 2017, 42, 130-140.

2 D. N. Woolfson, Subcell. Biochem., 2017, 82, 35-61.

3 I. Drobnak, H. Gradisar, A. Ljubetic, E. Merljak and R. Jerala, J. Am. Chem. Soc., 2017, 139, 8229-8236.

4 E. Lacroix, A. R. Viguera and L. Serrano, J. Mol. Biol., 1998, 284, 173-191.

5 J. R. Litowski and R. S. Hodges, J. Biol. Chem., 2002, 277, 37272-37279.

6 H. Gradišar and R. Jerala, J. Pept. Sci., 2011, 17, 100-106.

7 F. Thomas, A. L. Boyle, A. J. Burton and D. N. Woolfson, J. Am. Chem. Soc., 2013, 135, 5161-5166.

8 R. O. Crooks, A. Lathbridge, A. S. Panek and J. M. Mason, Biochemistry, 2017, 56, 1573-1584.

9 J. Yang, C. Xu, C. Wang and J. Kopeček, Biomacromolecules, 2006, 7, 1187-1195.

10 C. Aronsson, S. Danmark, F. Zhou, P. Oberg, K. Enander, H. Su and D. Aili, Sci. Rep., 2015, 5, 14063.

11 I. Tunn, A. S. de Léon, K. G. Blank and M. J. Harrington, Nanoscale, 2018, 10, 22725-22729.
12 A. L. Boyle, E. H. C. Bromley, G. J. Bartlett, R. B. Sessions, T. H. Sharp, C. L. Williams, P. M. G. Curmi, N. R. Forde, H. Linke and D. N. Woolfson, J. Am. Chem. Soc., 2012, 134, 15457-15467.

13 A. Ljubetic, F. Lapenta, H. Gradisar, I. Drobnak, J. Aupic, Z. Strmsek, D. Lainscek, I. Hafner-Bratkovic, A. Majerle, N. Krivec, M. Bencina, T. Pisanski, T. C. Velickovic, A. Round, J. M. Carazo, R. Melero and R. Jerala, Nat. Biotechnol., 2017, 35, 1094-1101.

14 H. Chao, D. L. Bautista, J. R. Litowski, R. T. Irvin and R. S. Hodges, J. Chromatogr. B: Biomed. Sci. Appl., 1998, 715, 307-329.

15 F. Thomas, W. M. Dawson, E. J. M. Lang, A. J. Burton, G. J. Bartlett, G. G. Rhys, A. J. Mulholland and D. N. Woolfson, ACS Synth. Biol., 2018, 7, 1808-1816.

16 M. Goktas, C. Luo, R. M. A. Sullan, A. E. Bergues-Pupo, R. Lipowsky, A. Vila Verde and K. G. Blank, Chem. Sci., 2018, 9, 4610-4621.

17 C. N. Pace and J. M. Scholtz, Biophys. J., 1998, 75, 422-427. 18 E. O'Shea, R. Rutkowski and P. Kim, Science, 1989, 243, 538-542.

19 J. M. Fletcher, G. J. Bartlett, A. L. Boyle, J. J. Danon, L. E. Rush, A. N. Lupas and D. N. Woolfson, ACS Chem. Biol., 2017, 12, 528-538.

20 A. Akmal and V. Muñoz, Proteins: Struct., Funct., Bioinf., 2004, 57, 142-152.

21 E. Evans and K. Ritchie, Biophys. J., 1997, 72, 1541-1555.

22 B. Ibarra-Molero, G. I. Makhatadze and C. R. Matthews, Biochemistry, 2001, 40, 719-731.

23 A. E. Bergues-Pupo, M. Goktas, I. Tunn, P. Lopez-Garcia, A. V. Verde, K. G. Blank and A. Valleriani, J. Chem. Phys., 2018, 149, 244120.

24 D. D. Root, V. K. Yadavalli, J. G. Forbes and K. Wang, Biophys. J., 2006, 90, 2852-2866.

25 S. G. Falkovich, I. M. Neelov and A. A. Darinskii, Polym. Sci., Ser. A, 2010, 52, 662-670.

26 Z. Qin and M. J. Buehler, Phys. Rev. Lett., 2010, 104, 198304.

27 P. Chakrabarti and D. Pal, Prog. Biophys. Mol. Biol., 2001, 76, 100-102.

28 A. Chakrabartty, T. Kortemme and R. L. Baldwin, Protein Sci., 1994, 3, 843-852.

29 A. E. Bergues-Pupo, K. G. Blank, R. Lipowsky and A. Vila Verde, Phys. Chem. Chem. Phys., 2018, 20, 29105-29115.

30 M. S. Cheung, A. E. García and J. N. Onuchic, Proc. Natl. Acad. Sci. U. S. A., 2002, 99, 685-690.

31 R. Zhou, X. Huang, C. J. Margulis and B. J. Berne, Science, 2004, 305, 1605-1609.

32 P. Liu, X. Huang, R. Zhou and B. J. Berne, Nature, 2005, 437, 159-162.

33 I. Popa, J. M. Fernandez and S. Garcia-Manyes, J. Biol. Chem., 2011, 286, 31072-31079.

34 A. K. Jana, J. C. Jose and N. Sengupta, Phys. Chem. Chem. Phys., 2013, 15, 837-844.

35 A. K. Jana and N. Sengupta, Biophys. J., 2012, 102, 1889-1896. 
36 R. C. Remsing, E. Xi and A. J. Patel, J. Phys. Chem. B, 2018, 122, 3635-3646.

37 D. P. Sadler, E. Petrik, Y. Taniguchi, J. R. Pullen, M. Kawakami, S. E. Radford and D. J. Brockwell, J. Mol. Biol., 2009, 393, 237-248.

38 T. Bu, H. C. Wang and H. Li, Langmuir, 2012, 28, 41 M. Goktas and K. G. Blank, Adv. Mater. Interfaces, 2017, 12319-12325.
39 D. J. Brockwell, G. S. Beddard, J. Clarkson, R. C. Zinober, A. W. Blake, J. Trinick, P. D. Olmsted, D. A. Smith and S. E. Radford, Biophys. J., 2002, 83, 458-472.

40 R. B. Best, S. B. Fowler, J. L. T. Herrera, A. Steward, E. Paci and J. Clarke, J. Mol. Biol., 2003, 330, 867-877.

4, 1600441. 